\title{
Stand-alone device for the electrolytic fabrication of scanning near-field optical microscopy aperture probes
}

\author{
C. Haumann, Ch. Pelargus, H. G. Frey, R. Ros, and D. Anselmettia) \\ Experimental Biophysics \& Applied Nanosciences, Bielefeld University, Universitaetsstrasse 25, D-33615 \\ Bielefeld, Germany \\ J. Toquant and D. W. Pohl \\ Institute of Physics, University of Basel, Klingelbergstrasse 82, CH-4056 Basel, Switzerland
}

(Received 30 June 2004; accepted 18 December 2004; published online 1 March 2005)

\begin{abstract}
Near-field optical applications require the fast, stable, and reproducible fabrication of scanning near-field optical microscopy (SNOM) aperture probes in the submicrometer range. We have developed a stand-alone device for the electrolytic etching of nanoapertures with an integrated current and optical transmission monitoring and control. Probes with an aperture ranging from 50 to $100 \mathrm{~nm}$ were reproducibly fabricated with great reliability. With these probes, high resolution SNOM images of $100 \mathrm{~nm}$ test patterns and single dye molecules (Rhodamine 6G in poly(vinyl alcohol)) are measured and presented. Not requiring a SNOM setup, the stand-alone device is not only inexpensive and compact, but also insensitive to external disturbances.
\end{abstract}

\section{INTRODUCTION}

The resolution achievable with scanning near-field optical microscopy (SNOM) is determined by the optical quality of the near-field probes. ${ }^{1-3}$ Although a great variety of alternative probes like cantilevers ${ }^{4,5}$ or apertureless scattering probes $^{6-8}$ were developed, the tapered optical fiber is still the most commonly used SNOM probe.

The techniques used so far to fabricate aperture probes often suffer from poor reproducibility, e.g., the widespread shadowing technique ${ }^{9}$ and aperture punching, ${ }^{1,10}$ or require expensive equipment like for focused ion beam (FIB) milling. ${ }^{11}$ Mulin et al. ${ }^{12}$ introduced a method to fabricate SNOM aperture probes by controlled all solid state electrolysis that was improved by Bouhelier et al. ${ }^{13}$ more recently. Using a solid electrolyte to etch the aperture, avoids the problem of meniscus formation of a liquid electrolyte ${ }^{14}$ and enables the reproducible and controlled fabrication of good quality near-field aperture probes. The electrolytic etching of nanoapertures combines the advantages of the shadowing technique (cheap, quick) with the high quality of FIB (defined apertures with flat rims). Additionally, coating the probes with silver instead of aluminum results in a distinct smoother surface. Furthermore, the use of our stand-alone device opens new applications of the near-field probes beyond SNOM imaging. ${ }^{15}$

\section{TIP FABRICATION}

As a probe we use "tube etched" 16 single-mode silica glass fibers (FS-SN-3224, 3M), which have a cladding diameter of $125 \mu \mathrm{m}$ and a core diameter of $4 \mu \mathrm{m}$. The etching process is very stable and self-controlled, because the fibers

\footnotetext{
${ }^{\text {a) }}$ Author to whom correspondence should be addressed; electronic mail: dario.anselmetti@physik.uni-bielefeld.de
}

are etched inside their acrylate jackets. The etching solution consists of an aqueous $40 \%$ hydrofluoric acid solution (HF) (pur. Fluka) at room temperature into which $1 \mathrm{~cm}$ of the fibers is dipped for $2 \mathrm{~h}$. In order to protect the rest of the fiber and the fiber mounts against the acid vapors, the HF solution is covered by a thin overlayer (about $0.5 \mathrm{~cm}$ ) of $\mathrm{N}$-octane. After the etching process is stopped, the protective coating is removed by dissolving it in $75^{\circ} \mathrm{C}$ hot sulfuric acid $\left(98 \% \mathrm{H}_{2} \mathrm{SO}_{4}\right.$, pur., Fluka) for $1 \mathrm{~h}$. The etched fiber tips are subsequently coated completely with a silver layer of about $350 \mathrm{~nm}$. A smaller amount of silver normally causes pinholes, which would reduce the optical resolution. The silver is thermally evaporated onto the rotating fibers to allow homogeneous film thickness deposition on the glass surface.

Aperture formation is performed by using the standalone setup described in Fig. 1. The setup is integrated into a small metal box, in order to protect the aperture formation from acoustic noise, disturbing electromagnetic fields, and background light. We have developed a separate scanning tunneling microscopy controller, which consists of the PIfeedback loop (without a logarithmic current amplifier) to regulate the tip-sample distance via monitoring the apparent current, combined with a photomultiplier tube detection unit (PMT) (H 5784, Hamamatsu). Not requiring an objective, the PMT measures directly the light intensity emitted from the aperture during the opening process.

The electrolytic cell consists of a thin platelet of a solid electrolyte, which serves as a ionic conductor for silver ions, and a silver counterelectrode evaporated onto the electrolyte (Fig. 1, closeup). The sharpened and silver coated optical fiber builds the positive electrode. The electrolyte we use is amorphous silver metaphosphate-iodide $\left(\mathrm{AgPO}_{3}: \mathrm{AgI}\right)$, because it has to fulfill requirements like high conductivity, transparency, and ease of fabrication. Silver iodide (AgI: No.:10217, Sigma Aldrich) and silver metaphosphate 


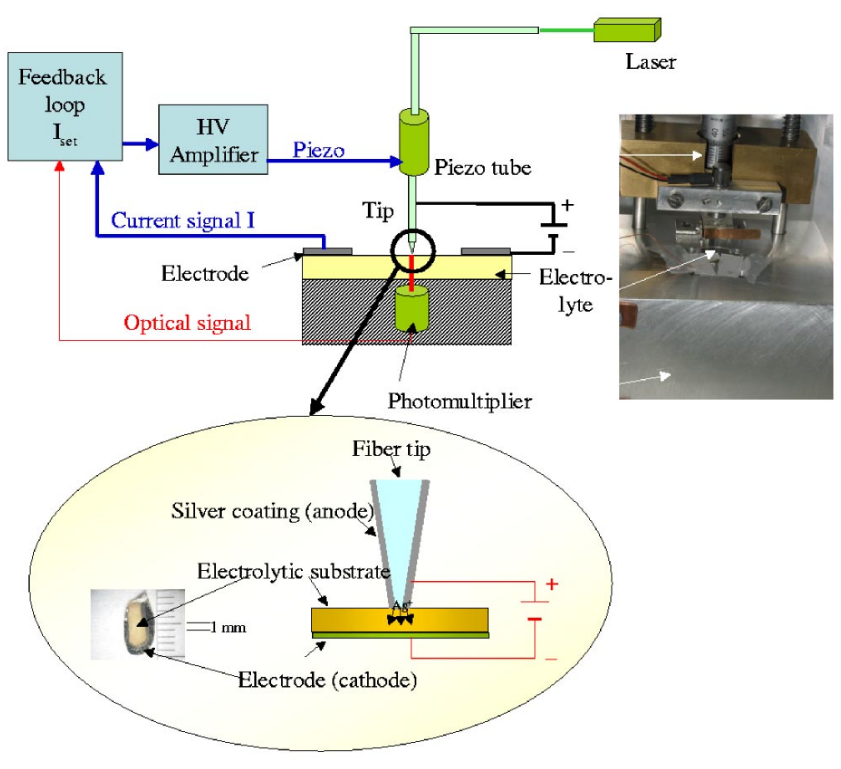

FIG. 1. (Color online) Aperture formation setup. The closeup shows the electrolytic cell in detail.

$\left(\mathrm{AgPO}_{3}\right.$ : Cas number: 13465-96-8, Citychemical) are mixed in an equimolar ratio to form a homogeneous powder. This powder is molten in a furnace at $650{ }^{\circ} \mathrm{C}$ for $45 \mathrm{~min}$. Subsequently one drop of the liquid mixture is poured onto a rotating cover slide in order to form a thin (about 1-2 mm), transparent, and bubblefree layer of the solid electrolyte. The silver electrode is deposited circularly onto the upper side of the electrolyte via thermal evaporation. During this process the middle is covered by a mask in order to conserve a tranparent part of the electrolyte. Through this part of the electrolyte, the light emitted from the generated aperture is detected by the PMT.

Laser light with a wavelength of $532 \mathrm{~nm}$ is coupled into the far end of the fiber probe with an overall length of about $1 \mathrm{~m}$. The probe tip is directly attached to the $z$-piezo tube (P-810.10, PI), which regulates the distance between the probe and the electrolyte after a coarse approach with a dc gear motor (M 1616 C, Faulhaber). The electrolytic cell is formed by connecting the probe to the positive and the counterelectrode to the negative pole of a voltage source. A bias voltage of $200 \mathrm{mV}$ is applied between the two electrodes. The feedback loop drives the piezo towards the surface until the setpoint of $I=10 \mathrm{pA}$ is reached. After contact the silver ions migrate via an adjacent waterfilm from the tip into the electrolyte. The process is stopped after the PMT measures a threshold intensity. Selection of the adequate threshold intensity determines the aperture size in the range from 50 to $100 \mathrm{~nm}$.

This stand-alone setup is of considerable advantage over the inclusion of the tip fabrication device into the SNOM, since the distance control is regulated via the apparent tunneling or point contact current, enabling a faster and more stable feedback regulation without the need of a lock-in amplifier. Therefore the aperture formation process is less sensitive to external disturbances such as vibrations, electromagnetic fields, or acoustic noise.
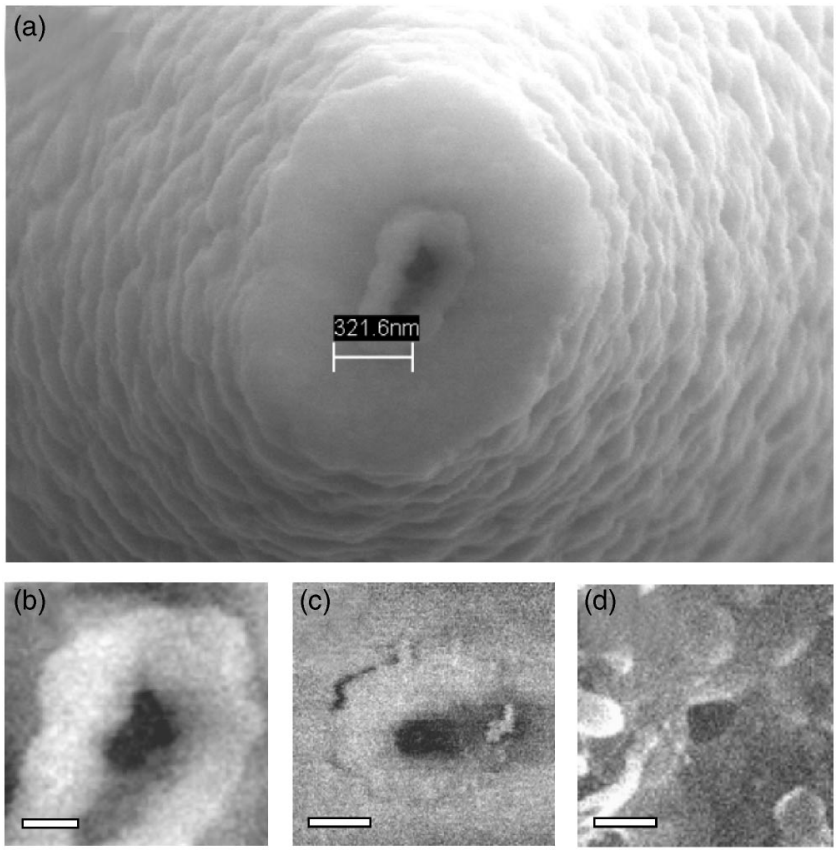

FIG. 2. Scanning electron micrographs of three routinely produced SNOM probes, with apertures $\leqslant 90 \mathrm{~nm}$ in diameter, surrounded by flat rims. (b) is a closeup of (a); (c) and (d) show the apertures of two additional probes. The scale bar of (b) to (d) corresponds to $100 \mathrm{~nm}$.

\section{RESULTS AND DISCUSSION}

Scanning electron microscope (SEM) images of three characteristic SNOM probes are shown in Fig. 2. The probes have typically flat ends with a diameter of $\sim 600 \mathrm{~nm}$. The dark spots in the center have diameters $<90 \mathrm{~nm}$ and represent the apertures.

The aperture probes were tested by using a SNOM scanstage (Triple-O) integrated into an inverted optical microscope (Zeiss Axiovert 100). Figure 3 gives an overview about our setup. Light of a diode pumped solid state laser

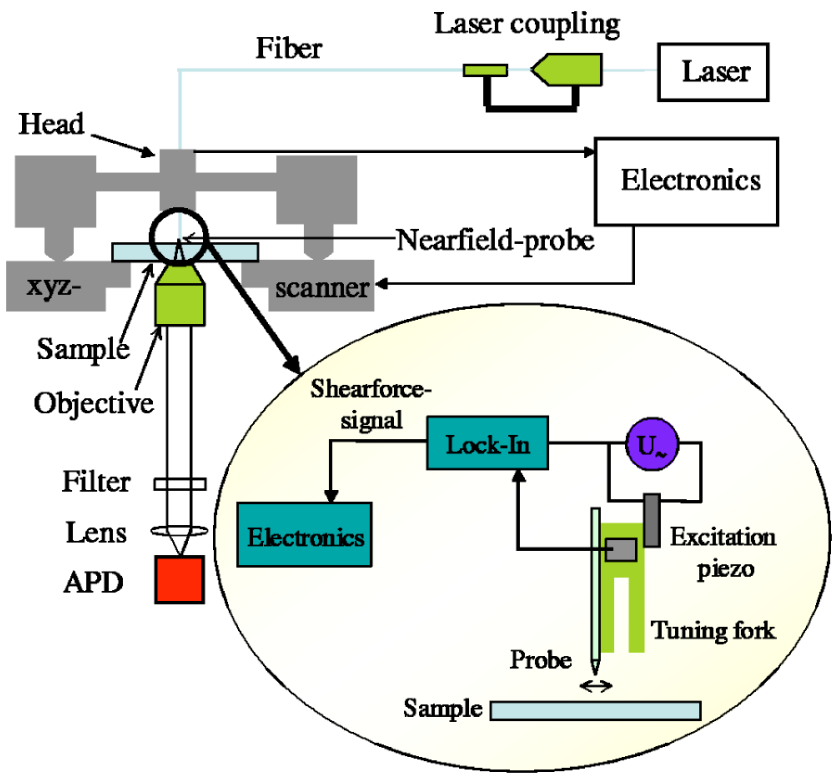

FIG. 3. (Color online) SNOM setup. A diode pumped solid state laser was used $(\lambda=532 \mathrm{~nm})$. SNOM scanstage is integrated into an inverted microscope. Distance control via shearforce detection. 


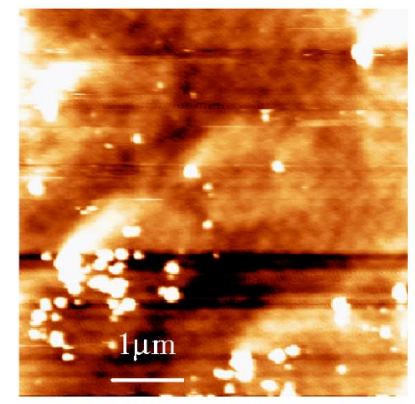

(a)

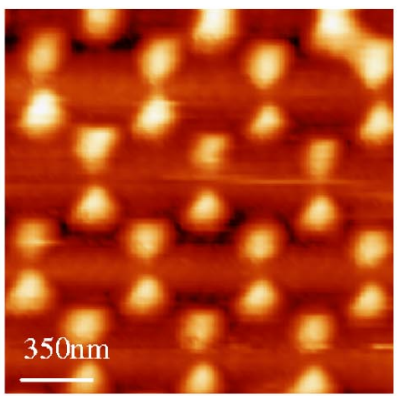

(c)

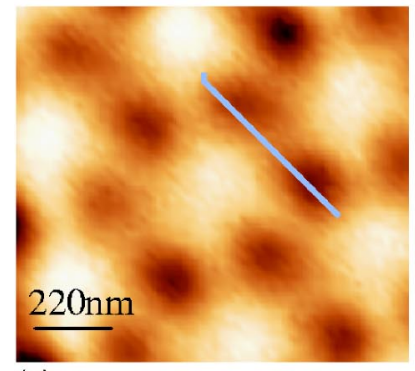

(e)

(f)

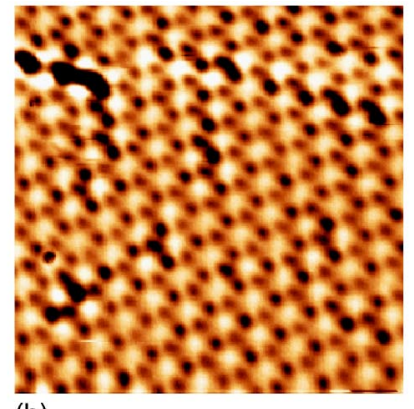

(b)

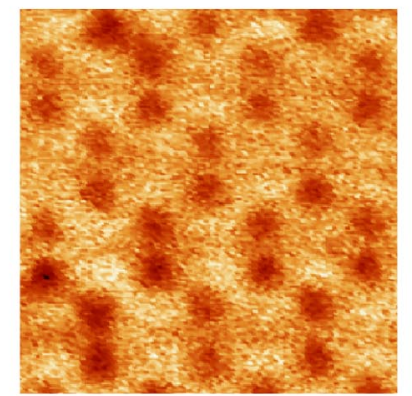

(d)

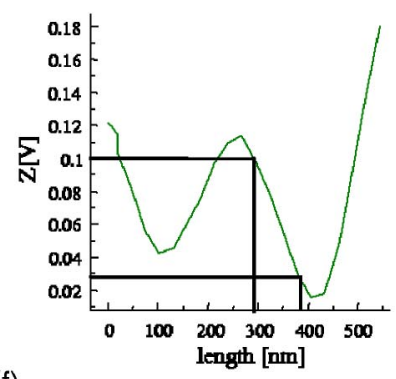

FIG. 4. (Color online) (a), (c) SNOM topographical and (b), (d) transmission images of an aluminum projection pattern. The aluminum islands appear bright in the topographical and dark in the transmission image. (e) Closeup of (b). (f) Associated cross section. The cross section was taken in the area marked by a solid line in (e). By applying a 10\%-90\% edge criteria, an optical resolution better than $90 \mathrm{~nm}$ was achieved.

( $\lambda=532 \mathrm{~nm}$, CrystaLaser) is coupled into the other end of the fiber. After interacting with the sample, the transmitted or fluorescent light is collected by an objective (CP-Achromat $40 \times$, N.A. $=0.65$, Zeiss) and focused onto an avalanche photodiode (APD) (SPCM-AQR-13, Perkin Elmer). To achieve an optimal detection efficiency, the probe is fixed at the optical axis of the system and the sample is scanned in $x, y$, and $z$ directions underneath the probe. The small sensitive area of the APD acts as a spatial filter and blocks all light which is not emitted from the focal plane.

The distance control is performed using a tuning fork as a shear force detector, where the fork is dithered by an excitation piezo. The resulting piezoelectric signal of the fork is measured with a lock-in amplifier and fed into a feedback loop to keep the shear force constant. ${ }^{17}$ The probe is glued to one prong of the tuning fork with a free end of about 0.5-1 mm. For fluorescence measurements a bandpass filter (HQ 575/50, AHF Analysentechnik) can be switched into the lightpath, to block the laser light.

In order to characterize the imaging capability of our device, we tested the electrolytically fabricated probes with
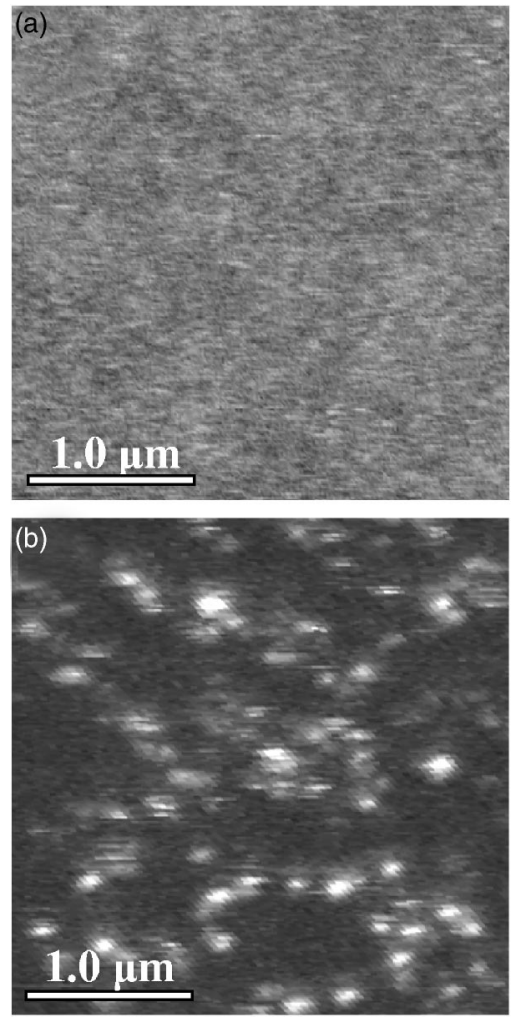

FIG. 5. SNOM topograph (a) with a simultaneously recorded fluorescence image (b) of single rhodamine $6 \mathrm{G}$ molecules in a polymer matrix.

an aluminum test pattern consisting of $\mathrm{Al}$ islands on glass fabricated by shadow evaporation of hexagonal close-packed $453 \mathrm{~nm}$ latex beads. ${ }^{18}$ Figure 4 shows the topographical (a), (c) and the corresponding transmission (b), (d) images of such a test sample. From the poor topographical resolution of image (a), one can infer that the optical image (b), showing a high resolution, was not influenced by the topography of the sample.

Some tips deliver not only a high topographical but also a good optical resolution, shown in Figs. 4(c) and 4(d), respectively. Here the topographical is even higher than the optical resolution and could be due to a small metal cluster on the very end of the tip. The high resolution shear force imaging capability can be regained by generating deliberately a small protrusion on the plateau of the tip end beside the aperture, by electron-beam deposition. ${ }^{19}$ Figure 4(e) shows a closeup of Fig. 4(b) with the associated cross section of the image Fig. 4(f). By applying a 10\%-90\% edge criteria $^{20}$ we achieve an optical resolution better than $90 \mathrm{~nm}$.

The electrolytically produced probes are also appropriate to investigate single fluorescent molecules. The system we investigated is the ionic dye rhodamine 6G (R6G, \# 83697, Fluka) in solid poly(vinyl alcohol) (PVA) (Merck). ${ }^{21} \mathrm{~A}$ solution of R6G in methanol (HPLC grade) is added to a $1 \mathrm{wt} \%$ solution of PVA $\left(M_{W}=7.2 \times 10^{4} \mathrm{~g} / \mathrm{mol}\right)$ in $1: 1$ methanolwater such that the concentration of R6G is $1 \mathrm{nM}$ with respect to the volume of the PVA. This solution is spin coated on a sonicated (successive acetone/ethyl alcohol/water) coverslide with $4000 \mathrm{rpm}$ for $30 \mathrm{~s}$.

Figure 5 shows a typical SNOM topograph (a) with a simultaneously recorded fluorescence image (b) of such a 
sample taken with our SNOM aperture probes. In the topography image no distinct feature can be identified as expected from a uniformly prepared and spincoated polymer film. Contrary, in the fluorescence image clearly resolved individual spots with a typical spot size of $100-150 \mathrm{~nm}$ can be identified. The spot size was measured at half maximum of each spot. Some of the spots exhibit noncontinuous characteristics, which are attributed to molecular blinking and photobleaching, clear signs of single molecule excitation phenomena. ${ }^{22}$

\section{ACKNOWLEDGMENTS}

The authors would like to thank A. Bouhelier and T. Röder for useful discussions and technical support and R. Eckel for the preparation of the R6G sample. SEM images and support from G. Reiss and co-workers are gratefully acknowledged.

${ }^{1}$ D. W. Pohl, W. Denk, and M. Lanz, Appl. Phys. Lett. 44, 651 (1984).

${ }^{2}$ U. Dürig, D. W. Pohl, and F. Rohner, J. Appl. Phys. 59, 3318 (1986).

${ }^{3}$ E. Betzig, J. K. Trautman, T. D. Harris, J. S. Weiner, and R. L. Kostelak, Science 251, 1468 (1991)

${ }^{4}$ E. Oesterschulze, O. Rudow, C. Mihalcea, W. Scholz, and S. Werner, Ultramicroscopy 71, 85 (1998).

${ }^{5}$ R. Eckert, J. M. Freyland, H. Gersen, H. Heinzelmann, G. Schürmann, W.
Noell, U. Staufer, and N. F. de Roij, J. Microsc. 202, 7 (2001).

${ }^{6}$ F. Zenhausern, M. P. Oboyle, and H. K. Wickramasinghe, Appl. Phys. Lett. 65, 1623 (1994).

${ }^{7}$ J. Koglin, U. C. Fischer, and H. Fuchs, Phys. Rev. B 55, 7977 (1997).

${ }^{8}$ A. Hartschuh, H. N. Pedrosa, L. Novotny, and T. D. Krauss, Science 301, 1354 (2003).

${ }^{9}$ G. A. Valaskovic, M. Holton, and G. H. Morrison, Appl. Opt. 34, 1215 (1995).

${ }^{10}$ T. Saiki and K. Matsuda, Appl. Phys. Lett. 74, 2773 (1999).

${ }^{11}$ J. A. Veerman, A. M. Otter, L. Kuipers, and N. F. van Hulst, Appl. Phys. Lett. 72, 3115 (1998).

${ }^{12}$ D. Mulin, D. Courjon, J.-P. Malugani, and B. Gauthier-Manuel, Appl. Phys. Lett. 71, 437 (1997).

${ }^{13}$ A. Bouhelier, J. Toquant, H. Tamaru, H.-J. Güntherodt, D. W. Pohl, and G. Schider, Appl. Phys. Lett. 79, 683 (2001).

${ }^{14}$ T. Saiki, S. Mononobe, M. Ohtsu, N. Saito, and J. Kusano, Appl. Phys. Lett. 68, 2612 (1996).

${ }^{15}$ A. P. Abel, M. G. Weller, G. L. Duveneck, M. Ehrat, and H. M. Widmer, Anal. Chem. 68, 2905 (1996).

${ }^{16}$ R. M. Stöckle, C. Fokas, V. Deckert, R. Zenobi, B. Sick, B. Hecht, and U. P. Wild, Appl. Phys. Lett. 75,160 (1999).

${ }^{17}$ K. Karrai and D. R. Grober, Appl. Phys. Lett. 66, 1842 (1995).

${ }^{18}$ U. C. Fischer and H. P. Zingsheim, J. Vac. Sci. Technol. 19, 881 (1981).

${ }^{19}$ H. G. Frey, F. Keilmann, A. Kriele, and R. Guckenberger, Appl. Phys. Lett. 81, 5030 (2002).

${ }^{20}$ T. Held, S. Emonin, O. Marti, and O. Hollricher, Rev. Sci. Instrum. 71, 3118 (2000).

${ }^{21}$ R. Zondervan, F. Kulzer, S. B. Orlinskii, and M. Orrit, J. Phys. Chem. A 107, 6770 (2003).

${ }^{22}$ W. E. Moerner and D. P. Fromm, Rev. Sci. Instrum. 74, 3597 (2003). 